INTERNATIONAL DESIGN CONFERENCE - DESIGN 2018

https://doi.org/10.21278/idc.2018.0375

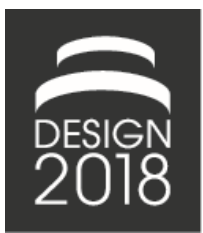

\title{
A COMPUTATIONAL APPROACH FOR COMBINATIONAL CREATIVITY IN DESIGN
}

\author{
L. Chen, P. Wang, F. Shi, J. Han and P. R. N. Childs
}

\begin{abstract}
Creativity is essential for innovative design. As an important topic in computational creativity research, how to model and generate creativity has sparked much interest. In this paper, combinational creativity is explored to provide potential semantic solutions and to generate visually synthesized solutions based on a given combination. This is achieved by a data-driven module and an artificial intelligence module respectively, which have been implemented as a creativity tool used in a design case study to demonstrate their effectiveness for assisting combinational creativity design.
\end{abstract}

Keywords: design creativity, computational design methods, artificial intelligence (AI), data driven design, combinational creativity

\section{Introduction}

Creativity is crucial for designing products and enabling innovation, and occurs through a process by which an agent uses its ability to generate ideas, solutions or products that are novel and valuable (Sarkar and Chakrabarti, 2011; Childs, 2018). To understand creativity in design, one common approach is to describe and understand the processes that generate potentially creative artefacts, which puts the focus on cognitive behaviours of a creative person or team (Maher, 2011). Researchers are also interested in modelling creativity in design based on their understanding of creativity processes, and try to define the properties of a computational system that is able to produce creative designs in a similar way, which is a part of computational creativity research (Besold et al., 2015).

With the explosion of information and the advance of data science, computational creativity has been a popular and promising field in creativity research. Not only has computational creativity been demonstrated to be capable of automatically producing art and poetry, but also it is able to tackle technical creative problems with creative problem solving (CPS) processes and generate creative ideas (also known as ideation) (Besold et al., 2015). As a well-known method of fostering creativity, combinational creativity has attracted researchers' attention on modelling it in a computational way. In this paper, we explore how to use the up-to-date data mining and artificial intelligence techniques to provide potential combinations and interpret combinations in a visual way.

\section{Combinational creativity}

By having observed a large number of examples of human creativity, Boden (2004) distinguishes three processes of creativity for modelling in artificial intelligence: exploratory creativity, transformational creativity and combinational creativity. In exploratory creativity, Boden views the exploration of conceptual spaces as a territory map exploration, in which all possibilities could be encompassed such as serendipity and creativity. Transformational creativity transforms the conceptual space to another space in new structures, such as transforming a real world picture to an image in Van Gogh's style. 
Combinational creativity, also known as conceptual combination, involves exploring creative combinations of two concepts or ideas. The "ideas" to be combined can be from arbitrary source, such as text, image, audio or video (Han et al., 2016).

As a natural process of human being's associative memory system, combinational creativity is the commonly used type of creativity and straightforward for a machine to achieve (Boden, 2009). It is also indicated by Frigotto and Riccaboni (2011) that combination is a nature of creativity by comparing generative and combinational creativity. Henriksen et al. (2014) also defined creativity as the process of making alterations to, and new combinations with, pre-existing ideas and artefacts, to produce something new.

Combinational creativity is also studied in the field of computational creativity. Wiggins (2006) summarises Boden's descriptive hierarchy of creativity, and proposes a framework for characterising computational creativity. Toivonen viewed data mining as a potentially creative activity which can be used by creative systems for combinational creativity (Toivonen and Gross, 2015). Arthur Koestler proposed a model of creative thinking which is referred to as bisociation. In Koestler's model, technical problems are solved by bridging knowledge in two otherwise not - or only very sparsely - connected domains whereas knowledge in a given domain is associated (Koestler, 1964). Of interest Dubitzky et al. compare bisociation with Boden's definition of combinational creativity and regards them as aligned with each other, and they proposed a framework for bisociation from the view of computational creativity based on their observations (Dubitzky et al., 2012).

Even though many methods have been proposed to understand and achieve combinational creativity, it is still challenging for machine to formulate and automatically produce it. As this research mainly focuses on noun-noun conceptual combination which is the conventional form of combinational creativity, the fist problem is to provide the combination space in which possible combinations can be found for given design task. The machine is required to find information which is not familiar for users but could be potentially combined to generate creativity. The following problem is the visual interpretation of such noun-noun textual combinations. For experienced designers, it may not be a problem for them to find solutions to how to visually interpret the combination of given base and additive. However, it could be elusive for a machine to "understand" the reason of doing this and even to generate reasonable visual combinations in which the value can be recognised (Boden, 2009).

In this paper, in order to solve the above two problems, a computational approach containing two modules is proposed. In the first module, a data-driven text mining method for implicit knowledge discovery is introduced. As a result, this module is able to provide far associations in a network graph so that users can use them to facilitate potential combinations. The second module proposes a supervised learning model to learn features of the base and additive of a pair of combination, and generate visual combinational design results.

\section{The computational approach for combinational creativity in design}

\subsection{Combinational creativity in design}

In product design, combinational creativity has been widely used in various forms. Bio-inspired design is a methodology dating back to the nineteen century which aims to obtain inspiration from biology and combine it with product design (Fu et al., 2014). Analogy is another form of combinational creativity involving the exploration of shared conceptual structure, according to Boden (2009). Design-by-analogy has been developed as a knowledge-based design methodology on how to identify analogies and analogous domains for analogous design, and the process of finding analogies can be viewed as the process of exploring possible combinations for combinational creativity design. Combinational creativity design can be classified into two categories, aesthetical combination and functional combination, as appearance and function are two main aspects of product design. The example shown in Figure 1 is an aesthetical combinational creativity design. In the example, the design solution is facilitated by combining the shape of feather and a normal table. In that combination case, either legs of a table is replaced with feather-like material, or the whole is made of feather-like material. This case can also be regarded as a bio-inspired design. The functional combination example is shown in Figure 2 , where a combinational creativity design is achieved by combining the function of a speaker with a 
shower head. According to Han et al. (2017), in a noun-noun combination, the first noun is the primary idea or the basic idea, which can be called the base. The second noun, which is the additional idea to be combined with, is named as the additive. For instance, in previous aesthetical combination case (in Figure 2), table is the base and feather is the additive.
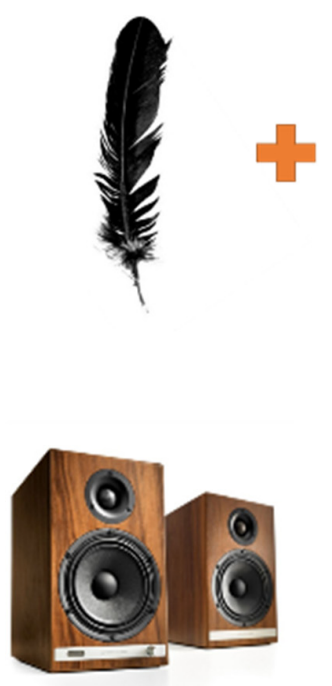

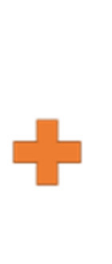

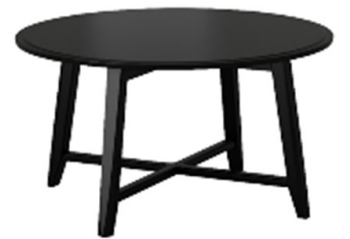

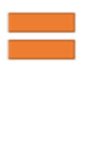

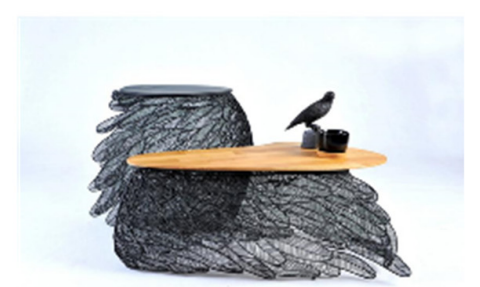

Figure 1. Aesthetical combination: feather + table

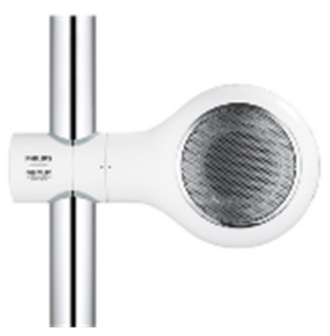

Figure 2. Functional combination: speaker + shower head

\subsection{The data-driven model for exploring possible combinations}

In the data-driven model, the problem of providing combination space is transformed to the exploration of cross-domain knowledge as those far associated concepts are more likely to be potential combinations such as analogies and metaphors (Chen et al., 2017). To facilitate such an implicit knowledge discovery process, a semantic network analysis and visualisation approach is proposed, as shown in Figure 3 . The first step is to collect a large amount of textual data which should be from various knowledge domains, which can be achieved with a web crawling method. As only concepts are involved in noun-noun combinational creativity, keywords are the exclusive data type in datasets, and this can be completed by applying statistical techniques, such as TF-IDF (Beliga, 2014). The collected datasets are then constructed in the structure of semantic network in the second step, where each concept is a vertex and the weight between two vertices has to be assigned. In order to capture far associations rather than close relationships between vertices in constructed network, global scaling and local fluctuation are applied to normalise the network (Shi et al., 2017a).

Our data-driven model provides two implicit knowledge retrieval methods to explore possible combinations, and they are "base" based search and "base and additive" based search respectively. Given the "base" of a combination, its corresponding far associated concepts which can be referred as potential "additives" can be retrieved by the first method. This method is achieved by applying Pythagorean means on the strength of explicit associations to evaluate the correlation degree of implicit association (Shi et al., 2017b). With known "base" and "additive" of an imagined combination, the second method is able to explain how this pair of concepts can be linked across a set of other concepts, which help users generate an idea of how to combine this imagined combination. This method is achieved by implementing the shortest path searching algorithms proposed by Shi et al. (2017b).

Results of "base" based searches and "base and additive" based searches can be shown in a semantic network graph. A semantic network is flexible allowing integration of a large amount of information from various domains with varying quality in a flexible way (Hao et al., 2014), and its structure provides a quantitative representation of the "interconnectedness" between vertices, such as the length of an edge and the size of a vertex. Furthermore, it allows users to explore and interact with retrieved data freely so that it gives users more chances to find possible combinations. 


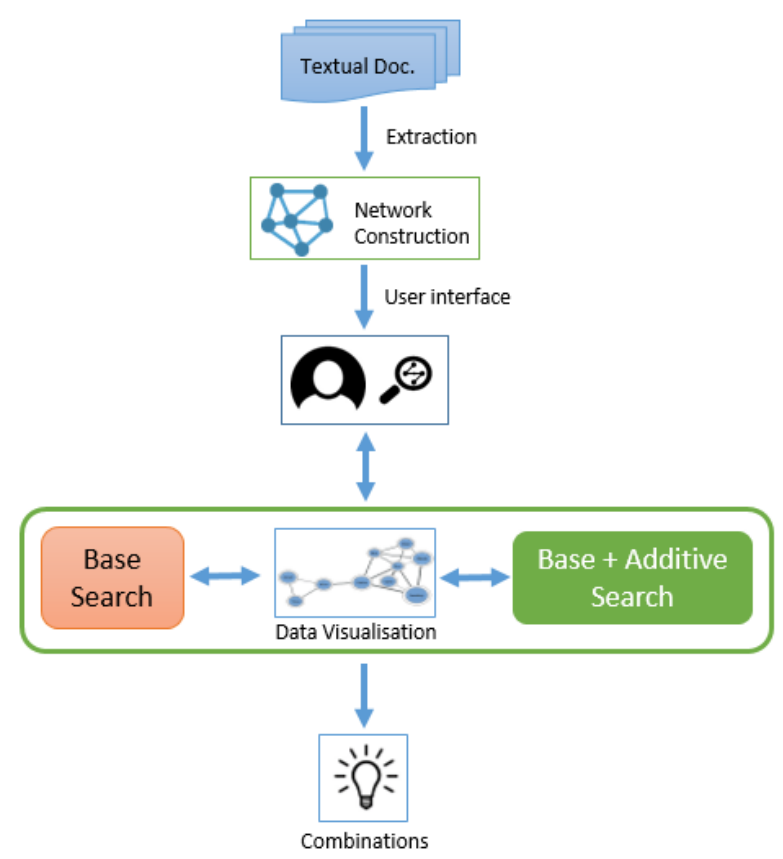

Figure 3. The data-driven module

\subsection{The artificial intelligence based model for synthesizing a combination}

Given a pair of noun-noun combination, it is quite challenging to generate a photo-realistic image which synthesizes both concepts in a reasonable way so that it interprets such a combinational creativity idea. Recently in computer vision, generating images which borrow features from different images has been a popular research topic and has made some promising achievements. Gatys et al. (2016) demonstrated how to use feature representations from high-performing Convolutional Neural Networks to transfer image style between arbitrary images. Wu et al. (2016) introduced a 3D Generative Adversarial Network (3D-GAN) to generate 3D chairs, tables and cars given specific object types, viewpoints and colour. Zhang et al. (2016) introduced stacked Generative Adversarial Networks (stack-GAN) to generate photo-realistic images based on textual description. Isola et al. (2016) investigated conditional adversarial networks for image-to-image translation problems, such as transforming the sketch of a bag to a well-fined colourful image.

Among current image generation models, Generative Adversarial Networks (GAN) are widely used and have shown great performance. The GAN was introduced by Goodfellow et al. (2014), and it basically consists of a generator $\mathrm{G}$ and a discriminator $\mathrm{D}$. The generator $\mathrm{G}$ is able to generate images randomly by sampling noise vectors from a distribution such as a Gaussian distribution. While the discriminator $\mathrm{D}$ takes an image either from the generator $\mathrm{G}$ or training datasets, and outputs the probability of the image to be real. The generator $\mathrm{G}$ is modelled to learn from the true data distribution by generating images that are difficult for the discriminator $\mathrm{D}$ to distinguish from real images, while the discriminator $\mathrm{D}$ is modelled to output a low probability when fed a "fake" image, and produce a high probability for a sample from true datasets. These two models are trained adversarially to compete with each other so that their capability can be improved.

Assuming there are images from two categories which represent two distinct concepts, the problem becomes how to generate images which synthesize both concepts so that a combinational creativity can be achieved. In this paper, we propose a model which contains one generator $\mathrm{G}$ and two discriminators $\mathrm{D}$ for neural networks training. Each of the discriminator is responsible for distinguishing one category's images from the images generated by the generator G. Given two datasets of images representing two known concepts, no pre-processing is required to be applied to train images but to keep them all in the same size. Then these images are taken by the model as two inputs, and are competed in two discriminators with images generated by Gaussian distribution in the generator G. Deep convolutional generative adversarial networks (DCGAN) (Radford et al., 2015) are applied to train the generator and two discriminators. For each discriminator, the last convolution layer is flattened and fed into a single sigmoid output. 


\section{Design case study}

\subsection{Design task}

To test our proposed approach of assisting generate combinational creativity in design, a design ideation case study was conducted. The design task was to find combinational design solutions for designing a spoon, and the only requirements are: the design spoon should be used to assist in eating soup; the more solutions the better. The task had two parts, one was to find semantic solutions using our proposed datadriven module, and another one was to generate visual solutions with proposed artificial intelligence based module. In the first part, participants were expected to generate ideas which combine "spoon" and other concepts by searching in provided datasets and exploring a network graph. For the second part, given "spoon" and "leaf", participants were asked to design a spoon which should be inspired from natural leaf. As this task was open-mind, the inspiration was allowed to be various, such as extracting features from leaf. Both parts follow the same requirements.

\subsection{Implementation of the creativity tool}

The creativity tool is developed as an integration of the two modules proposed. Its front-end interface was developed as a webpage in which users' search requests can be received and results can be shown as a network graph (for the data-driven module) and images (for the artificial intelligence module). The back-end engine was implemented with algorithms in Python, and the NetworkX library was used to store and search graph data in the data-driven module, while Tensorflow was utilized to implement proposed generative adversarial networks.

In the data-driven module, semantic data was collected from various resources in order to keep high diversity of established database. The data resources include design websites, such as blog-based Yanko design and prize-based red-dot, and Wikipedia for general knowledge collection. The textual content was captured with a web crawler, and then transferred to nouns and noun-phrases with natural language processing techniques such as tokenization and POS tagging in Stanford CoreNLP toolkit (Manning et al., 2014). These extracted concepts were used as vertices in the semantic ontology-based network, and the raw weights of edges were originally assigned based on the appearance of linked two vertices in the same sentence. As introduced in Section 3.2, normalisation was applied to the constructed network for implicit knowledge discovery, and Pythagorean means and shortest path searching algorithms were implemented as well for "base" and "base and additive" search respectively.

Before implementing the artificial intelligence based model, two training datasets were established containing 3772 images of spoons and 7408 images of leaves respectively. All images are kept in the size of $256 \mathrm{X} 256$. In the proposed model, the generator was trained as a deconvolutional network with 200 values of Gaussian distribution as input, using batch normalization and leaky-relu. Given two types of image $x_{A}$ and $x_{B}$ ("spoon" and "leaf" respectively in our case), and two discriminators for each of them, we optimized the following min-max problem:

$$
\begin{aligned}
\min _{G} \max _{D_{A} D_{B}} V\left(D_{A}, D_{B}, G\right) \\
\quad=\mathbb{E}_{x \sim p_{\text {data }}(x)}\left[\log D_{A}(x)\right]+\mathbb{E}_{x \sim p_{\text {data }}(x)}\left[\log D_{B}(x)\right] \\
+\mathbb{E}_{z \sim p_{Z}(z)}\left[\log \left(1-D_{A}(G(z))\right)\right. \\
\left.+\mathbb{E}_{z \sim p_{Z}(z)}\left[\log \left(1-D_{B}(G(z))\right)\right]\right]
\end{aligned}
$$

It was assumed that $x_{A}$ ("spoon") was the base image which could be similar to the product we expected to design, $x_{B}$ ("leaf") was the additive image which $x_{A}$ was expected to have its features. As these two types of images belong to different distributions, it is difficult for the generator to optimise its distribution, so the discriminators are more likely to converge quickly and lead to the failure of the generator. To solve this problem, three tricks were used:

- Batch normalization was not applied on discriminators.

- Soft and noisy labels on discriminators are used when inputting real images into them; we then replaced the labels of 1 to a random number between 0.7 and 1.2. This method is also well known as label smoothing. 
- We make the $x_{A}$ by transforming a RGB-based colourful image into a greyscale image, so the generator only learns the shape feature from $x_{A}$ without paying attention to the impact of colour on final combination results. This is because shape property of "spoon" is much more important than colour property in our design study.

For training, the Adam optimization approach is used with a momentum of 0.5. The learning rate is set to 0.002 with batch size of 128 and train for 300,000 iterations. Then the well trained generator $\mathrm{G}$ was used as a backend to randomly and automatically generate synthesized images which were regarded as the resource of combinational solutions to the design task.

\subsection{Implementation of the study}

The case study was conducted in two sessions, and each session lasted 10 minutes. Two groups of participants were recruited to complete the design task strictly on an individual basis. One group was required to use our developed tool to generate semantic solutions in the first session and visual solutions in the second session. Another group was asked to give solutions using natural brainstorming but was allowed to search information from online such as Google search engine, and all members have been familiar with natural brainstorming for idea generation. Each group had eight participants with design engineering background.

A brief introduction and explanation of the design task were given at the beginning of each session. The participants in the tool group were taught how to use our developed tool, while the participants of brainstorming group were provided with laptops for online information retrieval. In both sessions, each participant was given blank sheets to write down their design solutions including textual description and sketches. These sheets were collected at end of sessions, and interpreted in following data analysis section.

\subsection{Data analysis}

To estimate the combinational creativity of participants' design solutions, the Consensual Assessment Technique (CAT) was applied. This technique has been widely used for assessing creativity based on knowledgeable judge's intuitions about what metrics should be applied in order to measure specific creative work (Amabile, 1983). This is beneficial for tasks where it is difficult to set clear standards objectively. To apply this technique, generally two raters in minimum are required to rate every solution independently and then the level of their consensus will be evaluated. In our case study, both raters recruited were experts who have more than three years of experience in product design and also have experience of using CAT for creativity evaluation. Although the number of employed raters seems low, there is not a standard number of raters for a CAT0 assessment (Lai et al., 2006), and the number of expert raters is far less required than general raters (Achiche et al., 2013).

Both raters were blind of from which group each solution came, and there were 51 solutions generated from session 1 and 43 solutions generated in session 2. The raters are asked to score each solution within a scale from 1 to 7 (which means from the "least creative" to "most creative" in term of combinational creativity) based on their comprehension of combinational creativity, the requirements of the design task and comparisons between solutions. Several rounds were completed by the raters for estimating each session's solutions. For the first round, the raters were given all solutions placed in a random order, and were instructed to put these solutions into piles labelled from 1 to 7 . In the following rounds, as raters already had been familiar with the scale and all solutions, they rearranged all solutions repeatedly by moving solutions into different scales when needed until no more changes were made. Both sessions' rating tasks ended when raters thought all solutions had been well evaluated.

The consensus between two raters was evaluated based on Stemler's adjacent agreement (Stemler, 2004). For each rating task where a score within seven levels was given, consensus was considered to be reached if the score given by one rater did not differ by more than one point above or below the score given by another rater. As a consequence, the percentages of adjacent agreement between raters were $84 \%$ for session 1 and $81 \%$ for session 2 . To estimate the consistency between raters, Cohen's Kappa value was calculated as well. The Cohen's Kappa value was computed by transforming the scale from original seven levels to three levels. Specifically, scores of 1 and 2 fall into category "Low", score of 3 , 4 and 5 are in category "Normal", and score of 6 and 7 fall into category "High". As the results calculated 
for both sessions were 0.782 and 0.714 , which are greater than 0.50 , the rating work is typically considered as acceptable and reliable based on CAT.

\subsection{Results}

After the consensus check of two raters' results, the average of their scores for each rating was used in the statistical analysis. A normality check was conducted with Shapiro-Wilk's W test across two groups in two sessions. All the $\mathrm{p}$ values obtained are bigger than 0.05 , which means that our data distribution did not conform to normal distributions. Therefore, the independent Mann-Whitney U-test was chosen to interpret the coded data. In session 1 , the $\mathrm{p}$ value of the tool group is 4.9 which is significantly higher than 3.3 for the brainstorming group. Similarly, for the second session, the tool group performed significantly better than the brainstorming group ( $\mathrm{p}=4.1$ and $\mathrm{p}=2.9$, respectively), as shown in Figure 4 .

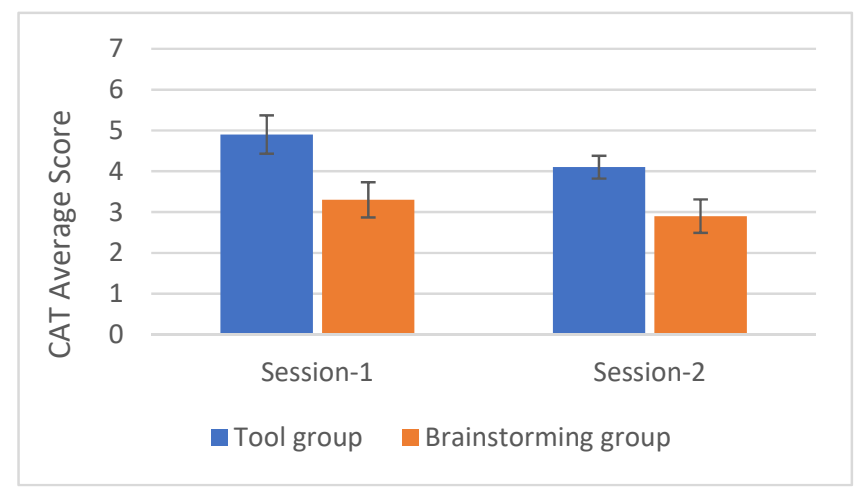

Figure 4. Results of CAT scores for combinational creativity

\subsection{Discussion}

From the analysis it was found in the first session that the solutions generated by the tool group have higher combinational creativity than the brainstorming group. This is probably due to the use of "base" search and "base and additive" search in the data-driven module. Within limited time, the module helped provide more effective inspirations than the brainstorming group. For example, when exploring "spoon" in the tool, a link of "spoon -> coffee -> drink -> straw" appeared as a part of a network graph, as shown in Figure 5. Then a solution of combining "spoon" and "straw" was generated, which was illustrated as shown in Figure 6. By checking participants' solution sheets, we also found that participants in the brainstorming group tended to use similar ideas as their solutions. One example is that a participant in the brainstorming group gave four solutions of combining a spoon with banana, palm, carrot and cat's claw respectively. In the tool group, benefiting from the high diversity of semantic network data retrieved from the data-driven module, the tool was able to show concepts from various domains, which gave good diversity of generated solutions.

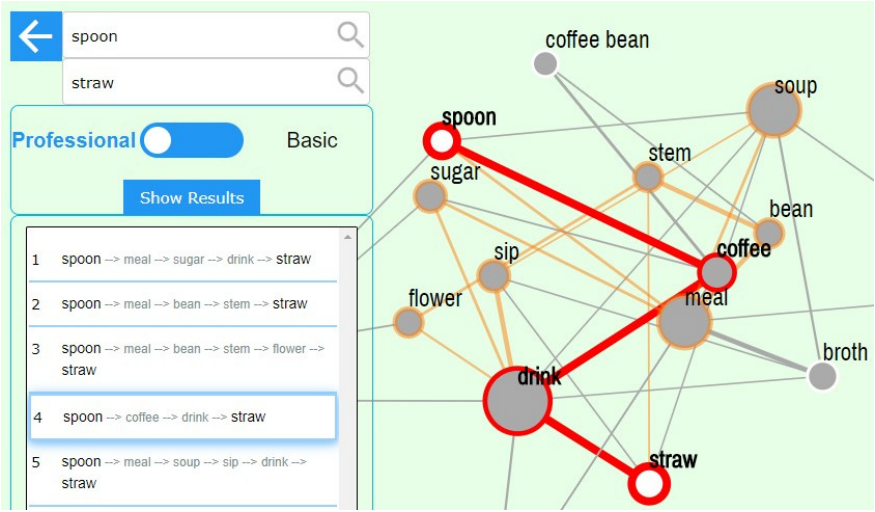

Figure 5. "straw" was found in the data-driven module 


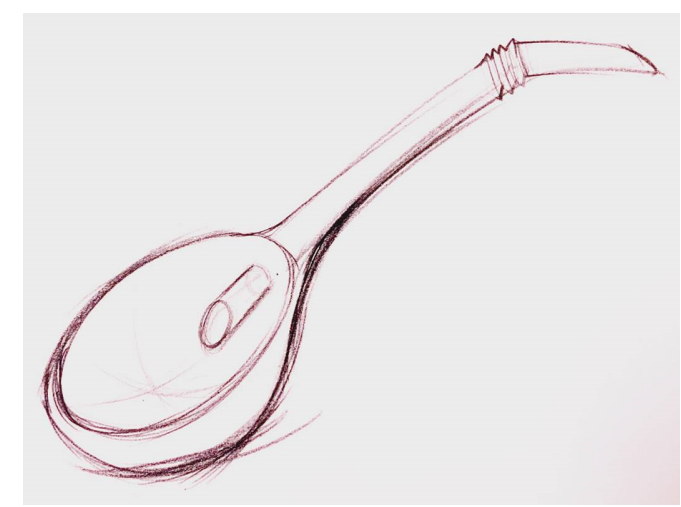

Figure 6. The illustration of a combined spoon with straw

Similarly, the artificial intelligence based module in the tool helped improve the performance of combinational creativity in the second session compared to the performance in the brainstorming group. Given the combination of "spoon" and "leaf", which can be regarded as a kind of combinational creativity, it seems more difficult for participants in the case study to find out more creative solutions. One easiest solution could be to design a spoon in a general leaf shape with green colour, which is the most popular case appeared in the brainstorming group. However, participants in the tool group were more likely to think beyond the easiest solution. For example, given the image shown in Figure 7, which was randomly and automatically generated by the artificial intelligence based model as it has been well trained to learn features from images of both "spoon" and "leaf", one participant perceived a special combination of "leaf" and "spoon" and came up with a novel solution of designing a leaf-like spoon with branches containing different seasoning, such salt and pepper, which was illustrated as shown in Figure 8.

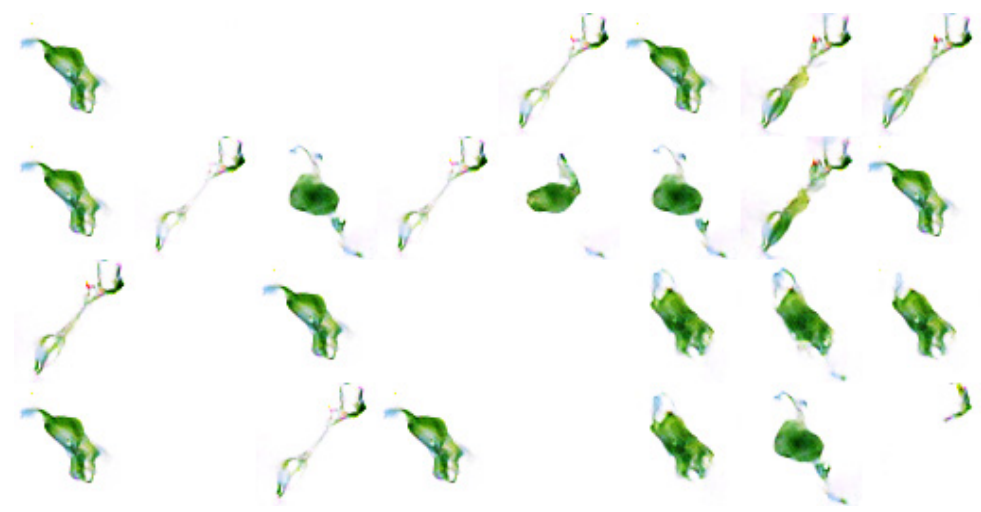

Figure 7. An image of leaf-inspired spoon generated by the tool

\section{Conclusion and future work}

To augment combinational creativity in design, a computational approach, which consists of a datadriven module and an artificial intelligence based module, is proposed in this paper. In the data-driven module, a semantic network graph can be retrieved by searching keywords or paths between keywords. This gives opportunities for users to explore possible combinations which can be used for combinational design, and it was demonstrated in session 1 of our case study that the tool group was able to generate higher rated combinational design solutions. Given the combinational design case of "spoon" and "leaf", our artificial intelligence based module was able to provide a variety of illustrations using the proposed deconvolutional generative adversarial networks algorithm. This module transforms a semantic combinational idea into a visual combinational design solution, and helps users think beyond semantic combinational cases. Although the proposed computational approach has been applied for design ideation in a case study, it could potentially be used for other general purposes in which idea generation activities are needed, such as advertising and commercial innovation for strategy. 


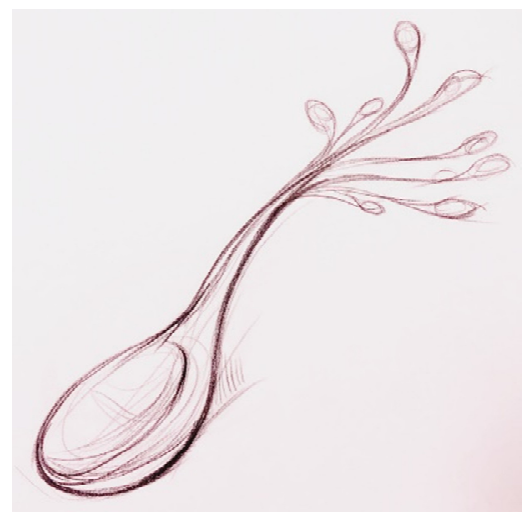

Figure 8. An illustration of leaf-inspired spoon

In our case study, due to the training process needed before running the artificial intelligence module, the data-driven module and artificial intelligence module have to work separately to support combinational creativity in design. This means that the process of transforming a semantic combination into a visual one is not coherent. Besides, we only trained the design case of "leaf" and "spoon" in the artificial intelligence module. In reality, arbitrary combinations may need to be generated, which requires a large database, much training time and computation resource. In the future, these issues may be solved with unsupervised learning method instead of the supervised learning algorithms proposed in this research.

\section{References}

Achiche, S., Appio, F.P., McAloone, T.C. and Di Minin, A. (2013), "Fuzzy decision support for tools selection in the core front end activities of new product development", Research in Engineering Design, Vol. 24 No. 1, pp. 1-18. https://doi.org/10.1007/s00163-012-0130-4

Amabile, T.M. (1983), “The social psychology of creativity: A componential conceptualization", Journal of Personality and Social Psychology, Vol. 45 No. 2, p. 357.

Beliga, S. (2014), Keyword Extraction: A Review of Methods and Approaches, University of Rijeka, Rijeka.

Besold, T.R., Schorlemmer, M. and Smaill, A. (2015), Computational Creativity Research: Towards Creative Machines, Atlantis Press, Paris. https://doi.org/10.2991/978-94-6239-085-0

Boden, M.A. (2004), The creative mind: Myths and mechanisms, Routledge Press.

Boden, M.A. (2009), "Computer models of creativity", AI Magazine, Vol. 30 No. 3, p. 23.

Chen, L., Shi, F., Han, J. and Childs, R.P. (2017), “A network-based computational model for creative knowledge discovery bridging human-computer interaction and data mining", International Design Engineering Technical Conferences \& Computers and Information in Engineering Conference (ASME IDETC/CIE), Cleverland, OH, USA, 2017.

Childs, P.R.N. (2018), Mechanical Design Engineering Handbook, 2nd ed., Butterworth-Heinemann.

Dubitzky, W., Kötter, T., Schmidt, O. and Berthold, M.R. (2012), "Towards Creative Information Exploration Based on Koestler's Concept of Bisociation", In: Berthold, M.R. (Ed.), Bisociative Knowledge Discovery. Lecture Notes in Computer Science, Vol. 7250. Springer, Berlin, Heidelberg, pp. 11-32. https://doi.org/10.1007/978-3-642-31830-6_2

Frigotto, M.L. and Riccaboni, M. (2011), "A few special cases: scientific creativity and network dynamics in the field of rare diseases", Scientometrics, Vol. 89 No. 1, pp. 397-420.

Fu, K., Moreno, D., Yang, M. and Wood, K.L. (2014), "Bio-Inspired Design: An Overview Investigating Open Questions From the Broader Field of Design-by-Analogy”, Journal of Mechanical Design, Vol. 136 No. 11, pp. 111102-18. https://doi.org/10.1115/1.4028289

Gatys, L.A., Ecker, A.S. and Bethge, M. (2016), "Image style transfer using convolutional neural networks", Proceedings of the IEEE Conference on Computer Vision and Pattern Recognition, 2016, pp. 2414-2423.

Goodfellow, I., Pouget-Abadie, J., Mirza, M., Xu, B., Warde-Farley, D. et al. (2014), "Generative adversarial nets", Advances in Neural Information Processing Systems, pp. 2672-2680.

Han, J., Park, D., Shi, F., Chen, L. and Childs, P. (2017), "Three driven approaches to combinational creativity", DS 87-8: Proceedings of the 21st International Conference on Engineering Design (ICED 17), Vol. 8: Human Behaviour in Design, Vancouver, Canada, August 21 - 25, 2017. 
Han, J., Shi, F. and Childs, P. (2016), "The combinator: a computer-based tool for idea generation", DS 84: Proceedings of the DESIGN 2016 14th International Design Conference, Dubrovnik, Croatia, May 16 - 19 , 2016, pp. 639-648.

Hao, J., Yan, Y., Gong, L., Wang, G. and Lin, J. (2014), "Knowledge map-based method for domain knowledge browsing", Decision Support Systems, Vol. 61, pp. 106-114. https://doi.org/10.1016/j.dss.2014.02.001

Henriksen, D., Mishra, P. and Deep-Play Research Group (2014), “Twisting knobs and connecting things: Rethinking Technology \& Creativity in the 21st Century", TechTrends, Vol. 58 No. 1, pp. 15-19.

Isola, P., Zhu, J.-Y., Zhou, T. and Efros, A.A. (2016), Image-to-image translation with conditional adversarial networks. [online] arXiv. Available at: https://arxiv.org/abs/1611.07004

Koestler, A. (1964), The act of creation, Hutchinson, England.

Lai, H.-H., Lin, Y.-C., Yeh, C.-H. and Wei, C.-H. (2006), "User-oriented design for the optimal combination on product design”, International Journal of Production Economics, Vol. 100 No. 2, pp. 253-267.

Maher, M.L. (2011), "Design creativity research: From the individual to the crowd", In: Taura, T. and Nagai, Y. (Eds.), Design Creativity 2010, Springer, London, pp. 41-47.

Manning, C.D., Surdeanu, M., Bauer, J., Finkel, J.R., Bethard, S. and McClosky, D. (2014), “The Stanford CoreNLP natural language processing toolkit", Proceedings of 52nd Annual Meeting of the Association for Computational Linguistics: System Demonstrations, Association for Computational Linguistics, pp. 55-60.

Radford, A., Metz, L. and Chintala, S. (2015), Unsupervised representation learning with deep convolutional generative adversarial networks. [online] arXiv. Available at: https://arxiv.org/abs/1511.06434

Sarkar, P. and Chakrabarti, A. (2011), “Assessing design creativity”, Design Studies, Vol. 32 No. 4, pp. $348-383$. https://doi.org/10.1016/j.destud.2011.01.002

Shi, F., Chen, L., Han, J. and Childs, P. (2017a), “A Data-Driven Text Mining and Semantic Network Analysis for Design Information Retrieval”, Journal of Mechanical Design, Vol. 139 No. 11, pp. 111402-14. https://doi.org/10.1115/1.4037649

Shi, F., Chen, L., Han, J. and Childs, P. (2017b), "Implicit knowledge discovery in design semantic network by applying Pythagorean means on shortest path searching", International Design Engineering Technical Conferences \& Computers and Information in Engineering Conference (ASME IDETC/CIE), Cleverland, OH, USA, August 6-9, 2017.

Stemler, S.E. (2004), “A comparison of consensus, consistency, and measurement approaches to estimating interrater reliability”, Practical Assessment, Research \& Evaluation, Vol. 9 No. 4, pp. 1-19.

Toivonen, H. and Gross, O. (2015), "Data mining and machine learning in computational creativity", Wiley Interdisciplinary Reviews: Data Mining and Knowledge Discovery, Vol. 5 No. 6, pp. 265-275. https://doi.org/10.1002/widm.1170

Wang, Z. (2013), Effects based stimulation of ideas in design and engineering, PhD thesis, Imperial College London.

Wiggins, G.A. (2006), "A preliminary framework for description, analysis and comparison of creative systems", Knowledge-Based Systems, Vol. 19 No. 7, pp. 449-458. https://doi.org/10.1016/j.knosys.2006.04.009

Wu, J., Zhang, C., Xue, T., Freeman, B. and Tenenbaum, J. (2016), "Learning a probabilistic latent space of object shapes via 3d generative-adversarial modeling", Advances in Neural Information Processing Systems, pp. 8290.

Zhang, H., Xu, T., Li, H., Zhang, S., Huang, X. et al. (2016), Stackgan: Text to photo-realistic image synthesis with stacked generative adversarial networks. [online] arXiv. Available at: https://arxiv.org/abs/1612.03242

Liuqing Chen, $\mathrm{PhD}$

Imperial College London, Dyson School of Design Engineering

Exhibition Road, SW7 1NA London, United Kingdom

Email: 1.chen15@imperial.ac.uk 